\title{
Influência das variáveis de processo na obtenção de argilas organofílicas
}

\section{(Influence of the process variables on obtaining organoclays)}

\author{
H. S. Ferreira ${ }^{1}$ L. F. A.Campos ${ }^{1}$, R. R. Menezes ${ }^{1}$, J. M. Cartaxo', \\ L. N. L. Santana ${ }^{2}$, G. A. Neves ${ }^{2}$, H. C. Ferreira ${ }^{2}$ \\ ${ }^{1}$ Departamento de Engenharia de Materiais, Universidade Federal da Paraíba, \\ Cidade Universitária, João Pessoa, PB \\ ${ }^{2}$ Unidade Acadêmica de Engenharia de Materiais, Universidade Federal de Campina Grande, \\ Campus I, Campina Grande, PB \\ hebersivini@ig.com.br,romualdomenezes@gmail.com,gelmires@dema.ufcg.edu.br, \\ heber@dema.ufcg.edu.br, lisiane@dema.ufcg.edu.br
}

\begin{abstract}
Resumo
As argilas bentoníticas, usadas como agente viscosificante e nanocompósitos, não podem ser usadas sem um prévio tratamento orgânico para que suas superfícies se tornem hidrofóbicas e possam ser dispersas em meios orgânicos. Estas argilas depois de tratadas são chamadas de argilas organofílicas, e são geralmente obtidas através da adição, em meio aquoso, de um agente organofilizante, geralmente um tensoativo, iônico, anfótero ou não iônico. O processo de organofilização, composto de várias etapas, nem sempre garante a obtenção de argilas organofílicas de boa qualidade, sendo importante destacar as variáveis de processo bem como a escolha das matérias-primas a serem utilizadas. Propõe-se então um estudo detalhado das variáveis envolvidas no processo de dispersão das argilas bentoníticas, e também no processo de organofilização procurando relacionar as viscosidades aparente e plástica das dispersões argilosas com a eficiência do processo de organofilização. O processo de organofilização será controlado pelos resultados de difração de raios X e termogravimetria. As variáveis de processo tanto envolvidas na dispersão das argilas quanto na organofilização, do ponto de vista da caracterização, não têm grande influência na incorporação do tensoativo às argilas bentoníticas, sendo influentes o tipo de argila e tensoativo e a presença de sódio como agente defloculante, o que é muito importante de vista industrial pela melhor operacionalidade das condições de processamento.
\end{abstract}

Palavras-chave: argilas organofílicas, tensoativos, variáveis de processos.

\begin{abstract}
The bentonitic clays, used as a thickening agent and nanocomposites, cannot be used without a prior treatment for their organic surfaces to become hydrophobic and be dispersed in organic media. These clays after treatment are called organoclays and are usually obtained through the addition of an organophilization agent, usually an ionic, amphoteric or nonionic surfactant. The process of organophilization is composed of several steps, and not always ensures the provision of good quality organoclays; it is important to highlight the process variables and the choice of raw materials to be used. It is here proposed a detailed study of the process variables involved in the dispersion of bentonitic clays, and also looking of the organophilization process related with the apparent and plastic viscosities of clay dispersions with the organophilization efficiency. The organophilization process will be controlled by observing the results of X-ray diffraction and thermogravimetry. It was observed through this study that both the process variables involved in the dispersion of the clays and in organophilization's view point characterization, have no great influence on the bentonite clay surfactant incorporation, and influence the clay type and surfactant and the presence sodium as dispersant agent, which is very important to the industrial serviceability of the best processing conditions.
\end{abstract}

Keywords: organoclays, surfactants, process variable.

\section{INTRODUÇÃO}

Bentonita pode ser definida como uma rocha constituída essencialmente por um argilomineral montmorilonítico (esmectítico), formado pela desvitrificação e subseqüente alteração química de um material vítreo, de origem ígnea, usualmente um tufo ou cinza vulcânica em ambientes alcalinos de circulação restrita de água.

As argilas organofílicas são constituídas basicamente por esmectitas e bentonitas que são modificadas com substancias orgânicas que apresentam afinidade química com sua estrutura cristalina e tornando-as hidrofóbicas. Uma vez tratadas, somente podem ser dispersas em meios orgânicos, não interagindo com água. Diversas rotas podem ser empregadas na modificação de argilas. Essas técnicas incluem: adsorção ou interações íon dipolo, troca de íons com cátions inorgânicos e complexos catiônicos, grafitização de compostos orgânicos, reação com ácidos, pilarização com cátions metálicos, polimerização interlamelar ou intra-partícula, desidroxilação e calcinação, delaminação e 
reagregação de argilominerais esmectíticos e tratamentos físicos como liofilização, ultra som e plasma $[1,2]$. Em aplicações específicas apenas o tratamento de organofilização não produz uma argila organofílica de qualidade, em virtude de diversos fatores que passamos a destacar: em primeiro lugar deve haver a escolha correta do tensoativo de forma a ser compatível com o meio dispersor; em segundo lugar deve haver a escolha de uma rota adequada de organofilização, destacando-se dentre estas a sistemáticas, a troca de cátions, onde o cátion $\mathrm{Na}^{+}$é substituído pelos radicais orgânicos e por último pelo fato das argilas naturais possuírem uma grande quantidade de elementos contaminantes, geralmente na forma de minerais acessórios [2]. Estes minerais não só dificultam a troca estequiométrica do tensoativo pelo íon $\mathrm{Na}^{+}$ como também prejudicam o desempenho das argilas [3, 4]. Devido aos problemas de obtenção de argilas organofílicas de boa qualidade foi desenvolvida uma sistemática de purificação de argilas esmectitas antes da organofilização, visando seu uso para diversos fins industriais [5]. Para avaliação da afinidade da argila organofilica com um meio orgânico dispersor, utiliza-se uma variante do tradicional inchamento de Foster [6], acoplado ao uso da difração de raios X para, através da expansão interplanar basal, maximizar a intercalação dos tensoativos, aprimorando assim o processo de organofilização. Em 2006 foi estudada a eliminação de matéria orgânica e ferro livre de bentonitas o que melhorou a incorporação de sais de amônio entre as lamelas de argilas [7]. A organofilização de argilas é tradicionalmente efetuada através da técnica de troca de íons, de forma mais detalhada, consiste basicamente em dispersar a argila em água quente, adicionar o sal quaternário de amônio previamente dissolvido e manter em agitação sob determinado período, lavar para retirar o excesso de sal que não reagiu, filtrar, secar e desagregar o material obtido [4]. Como pode ser verificado, diversas variáveis de processo estão envolvidas na dispersão da argila em água e da subseqüente organofilização, dentre elas podemos citar preparação da dispersão, velocidade de preparação da dispersão, cura da dispersão, presença de defloculante, temperatura da organofilização e seu tempo de cura. Outras variáveis que influenciam na organofilização e que não podem ser classificadas como de processo são: tipo de argila, tipo de tensoativo, etc. O estudo das variáveis de processo do ponto de vista industrial é da máxima importância, pois todo processamento industrial deverá ser efetuado em condições de processamento extremamente simples envolvendo operações unitárias que exijam equipamentos de baixo custo, simplicidade, facilidade de execução e reduzido custo operacional. Dentro desta ótica procuramos analisar todas as etapas do processo de organofilização desde a preparação das matérias primas até o produto final, visando a execução do processo de organofilização em escala industrial de forma a facilitar o seu processo de execução, maximizando sua produtividade.

Este trabalho tem por objetivo a otimização do processo de organofilização de argilas bentoníticas visando seu uso para fins industriais, através do estudo detalhado das variáveis de processo envolvidas na dispersão das argilas bentoníticas (velocidade de agitação, tempo e temperatura de cura), no processo de organofilização (tempo e temperatura de cura).

\section{MATERIAIS E MÉTODOS}

Foram utilizadas três amostras de argila bentoniticas, a chocolate, argila naturalmente policatiônica extraída de Boa Vista, PB, a Brasgel PA, argila sódica industrial proveniente da Bentonit União Nordeste e a closite NA+, argila sódica purificada, industrializada proveniente da empresa "Southern Clay", Gonzáles,TX, EUA. Os sais orgânicos utilizados foram Praepagem WB (cloreto de diestearil dimetil amônio, com 75\% de matéria ativa), e Praepagem HY (cloreto de alquil hidroxietil dimetil amônio, com $40 \%$ de matéria ativa).

A caracterização das amostras de argilas e das organofílicas foi efetuada por meio das seguintes técnicas: capacidade de troca de cátions (CTC) [8]; área específica (AE) [8]; análise granulométrica por difração de laser (AG) (equipamento Cilas 1064); análise química por fluorescência de raios $X$ (EDX 720 Shimadzu); difração de raios $X$ (DRX) (6000 Shimadzu); análise termogravimétrica (ATG) e análise térmica diferencial (ATD) (sistema de análise térmica Shimadzu TA 60H).

$\mathrm{Na}$ preparação utilizou-se uma dispersão com

Tabela I - Variáveis estudadas para dispersão e organofilização.

[Table I - Dispersion and organophilization variables studied.]

\begin{tabular}{|c|c|c|c|c|c|c|c|c|c|}
\hline 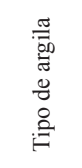 & 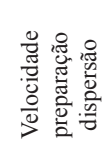 & 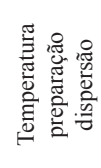 & 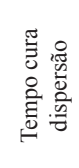 & 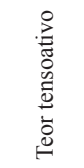 & 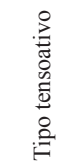 & 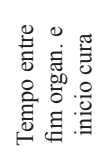 & 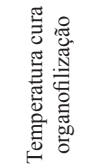 & 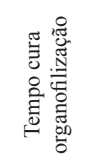 & 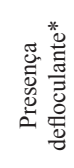 \\
\hline $\begin{array}{c}\mathrm{B}^{1} \\
\mathrm{CL}^{2} \\
\mathrm{C}^{3}\end{array}$ & $\begin{array}{c}1500 \\
17000 \\
(\mathrm{rpm})\end{array}$ & $\begin{array}{c}\mathrm{TA}^{4} \\
80^{\circ} \mathrm{C}\end{array}$ & $\begin{array}{c}0 \mathrm{~h} \\
24 \mathrm{~h}\end{array}$ & $60 \%$ & $\begin{array}{l}\mathrm{HY}^{5} \\
\mathrm{WB}^{6}\end{array}$ & $\mathrm{Oh}$ & $\begin{array}{c}\mathrm{E}^{6} 0^{7} \\
\mathrm{TA}^{4}\end{array}$ & $\begin{array}{l}24 \mathrm{~h} \\
96 \mathrm{~h}\end{array}$ & $\begin{array}{l}\mathrm{CD}^{8} \\
\mathrm{SD}^{9}\end{array}$ \\
\hline
\end{tabular}

1- Brasgel, 2 -Cloisite $\mathrm{Na}^{+}, 3$-Chocolate, 4-Temperatura Ambiente, 5 -Praepagem HY, 6 - Praepagem WB, 7 - Estufa à $60^{\circ} \mathrm{C}, 8-$ Com defloculante, 9 -Sem defloculante, apenas Chocolate. 
concentração de 4,16\% em massa de argila bentonítica em água deionizada, e a seguir foram estudadas as variáveis de processo: temperatura de preparação, (temperatura ambiente (TA) e $80^{\circ} \mathrm{C}$ ), isto é a temperatura da água antes da adição da argila, que tem sua importância fundamentada no fato de que, sob influência do calor, a hidratação das argilas ocorre de maneira mais rápida que em temperatura ambiente, permitindo obtenção de um comportamento reológico adequado em menor tempo, velocidade de preparação, (1500 rpm e $17000 \mathrm{rpm})_{2}$ isto é, a velocidade do agitador durante os 20 min necessários para que a argila se disperse na água, que tem influência na energia disponível para agitar o sistema, promover a hidratação da argila e destruir os aglomerados já existentes e os novos formados pela presença da água e tempo de cura, $(0 \mathrm{~h}$ e $24 \mathrm{~h})$, que se refere ao tempo que a dispersão permanece em repouso antes da organofilização, que pode influenciar no tempo necessário para completa hidratação da argila para as demais etapas. Cada dispersão foi agitada e o tensoativo iônico foi sendo adicionado aos poucos com o teor de 60\% [9] em massa em relação ao teor de argila seca, a agitação foi mantida por 20 min. Após a agitação verificou-se a influência do tempo de cura da organofilização ( 24 h e 96 h) e da temperatura de cura (TA e $60^{\circ} \mathrm{C}$ ) e em seguida a argila já organofílica foi submetida à filtração à vácuo, lavando-se com água deionizada, após esta etapa a torta foi seca em estufa a $60^{\circ} \mathrm{C}$ por aproximadamente $48 \mathrm{~h}$.

A amostra chocolate foi organofilizada com e sem a presença de defloculante sódico (CD e SD), a fim de se verificar a necessidade deste tipo de tratamento corriqueiro no processo de beneficiamento de bentonitas.

Foi também efetuada uma correlação entre as viscosidades aparente e plástica das dispersões argilosas, tanto as industriais como a natural, com os resultados de organofilização, visando encontrar uma relação entre a reologia e a eficiência da organofilização.

A Tabela I apresenta, de forma resumida, todas as variáveis estudadas.

O controle do processo de organofilização foi efetuado por DRX e por ATG, que reconhecidamente é o melhor método de avaliação do processo de organofilização tendo em vista que a quantidade de tensoativo intercalado é determinada. O uso da DRX acima mencionado constituise num método qualitativo, e verificaremos se há alguma correlação entre a expansão da distancia interplanar $d_{001} e$ quantidade de tensoativo intercalada, como já mencionado, determinada por ATG.

\section{RESULTADOS E DISCUSSÃO}

Na Tabela II estão apresentados os resultados da CTC e $\mathrm{AE}$ das argilas estudadas.

As amostras industrializadas, por serem sódicas, apresentaram maior capacidade de adsorver o azul de metileno. A CTC da argila chocolate apresentou-se dentro dos valores típicos de argilas não sódicas e as demais, valores típicos de argilas bentoníticas sódicas [10-12]. Todos os valores apresentaram-se dentro dos valores típicos de argilas bentoníticas. Os valores de AE confirmam os valores obtidos no ensaio de CTC, onde a maior CTC representa uma maior AE [10].

Na Tabela III está apresentada a composição química das argilas estudadas.

O teor de $\mathrm{SiO}_{2}$ é similar em todas as amostras de argilas; para o teor de $\mathrm{Al}_{2} \mathrm{O}_{3}$, as argilas chocolate e Brasgel PA apresentaram teores similares, já a cloisite $\mathrm{Na}^{+}$apresentou um teor mais elevado, evidenciando a maior concentração da fração argila. Para o teor de $\mathrm{Fe}_{2} \mathrm{O}_{3}$, observaramse grandes diferenças, as argilas chocolate e Brasgel apresentaram elevado teor, já a cloisite $\mathrm{Na}^{+}$apresentou um teor significativamente menor; essa redução devese provavelmente ao processo de industrialização das amostras Brasgel e Cloisite $\mathrm{Na}^{+}$. Para o teor de $\mathrm{CaO}$ as argilas chocolate e Cloisite $\mathrm{Na}^{+}$apresentaram baixo teor, a Brasgel apresentou um teor mais elevando evidenciando a origem geológica policatiônica da argila. Os teores de $\mathrm{MgO}$ apresentaram-se similares. Para o teor de $\mathrm{Na}_{2} \mathrm{O}$ observa-

Tabela II - Capacidade de troca de cátions e área especifica das argilas estudadas.

[Table II - Clay samples cation exchange capacity and surface area.]

\begin{tabular}{ccc}
\hline Amostra & $\begin{array}{c}\text { Capacidade de } \\
\text { troca de cátions } \\
(\mathrm{meq} / 100 \mathrm{~g})\end{array}$ & $\begin{array}{c}\text { Área específica } \\
\left(\mathrm{m}^{2} / \mathrm{g}\right)\end{array}$ \\
\hline Chocolate & 56 & 437,04 \\
Brasgel PA & 72 & 561,90 \\
Cloisite $\mathrm{Na}^{+}$ & 92 & 718,00 \\
\hline
\end{tabular}

Tabela III - Composição química das argilas estudadas. [Table III - Chemical composition of clays.]

\begin{tabular}{|c|c|c|c|c|c|c|c|c|c|}
\hline $\begin{array}{l}\text { Amostras } \\
\\
\text { Óxidos }\end{array}$ & $\begin{array}{l}\mathrm{SiO}_{2} \\
(\%)\end{array}$ & $\begin{array}{l}\mathrm{Al}_{2} \mathrm{O}_{3} \\
(\%)\end{array}$ & $\begin{array}{c}\mathrm{Fe}_{2} \mathrm{O}_{3} \\
(\%)\end{array}$ & $\begin{array}{l}\mathrm{CaO} \\
(\%)\end{array}$ & $\begin{array}{l}\mathrm{MgO} \\
(\%)\end{array}$ & $\begin{array}{l}\mathrm{Na}_{2} \mathrm{O} \\
(\%)\end{array}$ & $\begin{array}{l}\mathrm{K}_{2} \mathrm{O} \\
(\%)\end{array}$ & $\begin{array}{c}\text { Outros óxidos } \\
(\%)\end{array}$ & $\begin{array}{l}\text { PR } \\
(\%)\end{array}$ \\
\hline Chocolate & 59,77 & 17,31 & 10,37 & 0,30 & 2,21 & - & 0,21 & 1,07 & 8,72 \\
\hline Brasgel PA & 62,33 & 16,45 & 7,76 & 1,26 & 2,09 & 1,65 & 0,52 & 1,18 & 6,61 \\
\hline Cloisite $\mathrm{Na}^{+}$ & 60,14 & 23,34 & 4,10 & 0,40 & 2,05 & 2,37 & 0,03 & 0,71 & 6,82 \\
\hline
\end{tabular}

PR - Perda ao Rubro determinada por calcinação das amostras secas à $110{ }^{\circ} \mathrm{C}$ 
se que a argila chocolate, de natureza policatiônica, não apresentou o sódio na sua composição; já as argilas Brasgel e Cloisite $\mathrm{Na}^{+}$, a primeira tornada sódica industrialmente e a segunda naturalmente sódica, apresentaram o sódio em sua composição [10]. $\mathrm{O} \mathrm{K}_{2} \mathrm{O}$ e $\mathrm{CaO}$ e outros óxidos apresentaram-se em baixos teores. A PR apresentou valor compatível com os reportados [10-13]. De maneira global verificou-se que a argila possui composição química típica das argilas bentoníticas [10-13].

Na Fig. 1 estão apresentados os resultados de DRX das argilas estudadas.

Analisando-se o difratograma da Fig. 1 (chocolate), observou-se: presença do argilomineral esmectitico, caracterizado pela distância interplanar de 17,42 ̊̊ e 4,47 ̊̊, presença de
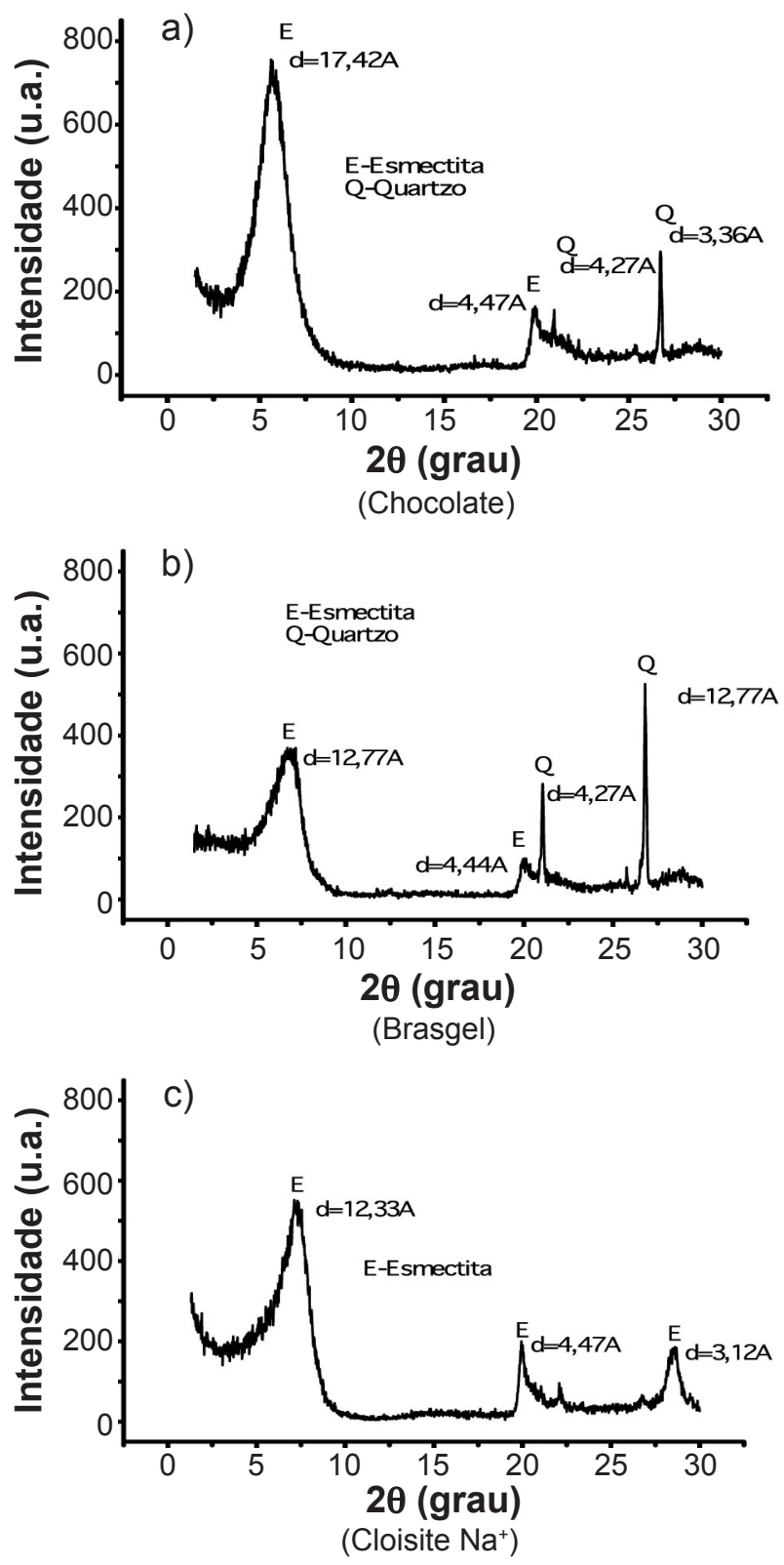

Figura 1: Difratogramas de raios $\mathrm{X}$ das argilas. [Figure 1: X-ray diffraction patterns of the clays.] quartzo, caracterizado pelas distâncias interplanares de $4,27 \AA$ e $3,36 \AA$; presença do argilomineral esmectitico, caracterizado pela distância interplanar de 12,77 $\AA$ e 4,44 $\AA$ presença de quartzo, caracterizado apenas pelas distâncias interplanares 4,27 ̊ e 3,36 ̊. No difratograma da Fig. 1 (cloisite) verificou-se a presença do argilomineral esmectitico, caracterizada pelas distâncias interplanares $12,33 \AA$, 4,47 ̊ e 3,12 ̊. De maneira global verificou-se que as argilas possuem difratogramas típicos das argilas
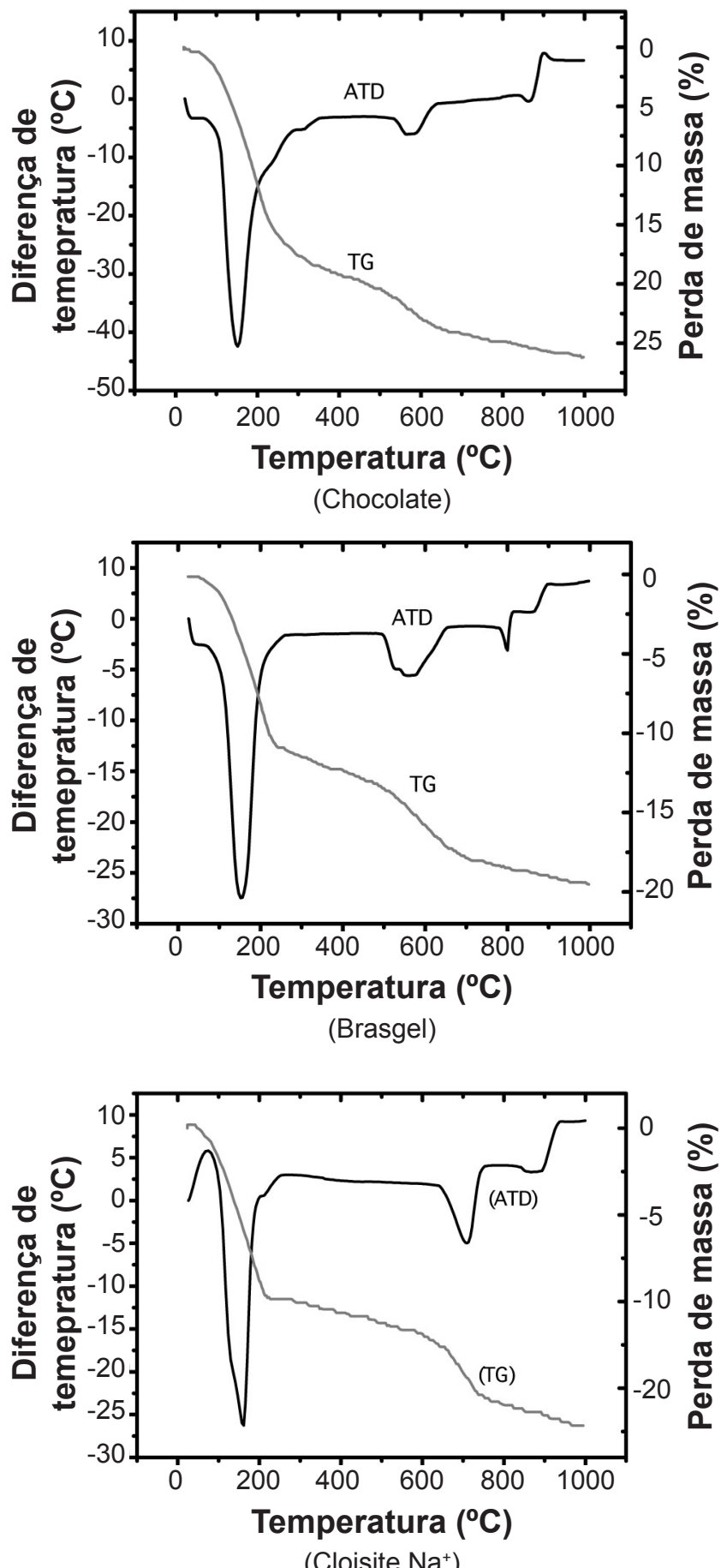

Figura 2: Analises térmicas das argilas estudadas.

[Figure 2: Thermal analysis of clays.] 
Tabela IV - Resultados de viscosidades das argilas estudadas.

[Table IV - Viscosities results of clays.]

\begin{tabular}{|c|c|c|c|c|c|c|c|}
\hline \multicolumn{2}{|c|}{$\begin{array}{c}\text { Amostra de } \\
\text { Argila }\end{array}$} & \multirow{2}{*}{$\begin{array}{c}\begin{array}{c}\text { Temperatura } \\
\text { da dispersão } \\
\left({ }^{\circ} \mathrm{C}\right)\end{array} \\
\text { TA }\end{array}$} & \multirow{2}{*}{$\begin{array}{c}\begin{array}{c}\text { velocidade } \\
\text { de agitação } \\
\text { (rpm) }\end{array} \\
1500\end{array}$} & \multirow{2}{*}{$\begin{array}{c}\text { tempo de } \\
\text { cura (h) } \\
0\end{array}$} & \multirow{2}{*}{$\frac{\mathrm{VA}(\mathrm{cP})}{4,5}$} & \multirow{2}{*}{$\begin{array}{c}\mathrm{VP}(\mathrm{cP}) \\
4,0\end{array}$} & \multirow[t]{2}{*}{$\begin{array}{l}\text { Qualidade } \\
\text { do resultado }\end{array}$} \\
\hline \multirow{8}{*}{\multicolumn{2}{|c|}{ 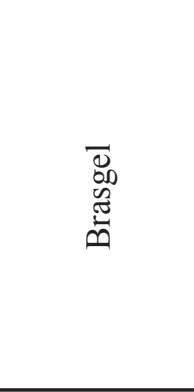 }} & & & & & & \\
\hline & & $\mathrm{TA}$ & 1500 & 24 & 6,5 & 3,0 & \\
\hline & & $\mathrm{TA}$ & 17000 & 0 & 7,5 & 5,0 & \\
\hline & & $\mathrm{TA}$ & 17000 & 24 & 8,5 & 4,5 & 3 \\
\hline & & 80 & 1500 & 0 & 5,75 & 4,5 & \\
\hline & & 80 & 1500 & 24 & 8,0 & 4,0 & 4 \\
\hline & & 80 & 17000 & 0 & 12,75 & 5,0 & 1 \\
\hline & & 80 & 17000 & 24 & 11,5 & 10,5 & 2 \\
\hline \multirow{8}{*}{\multicolumn{2}{|c|}{ 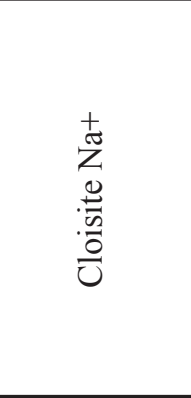 }} & TA & 1500 & 0 & 14,0 & 12,5 & 2 \\
\hline & & $\mathrm{TA}$ & 1500 & 24 & 8,75 & 8,0 & 4 \\
\hline & & TA & 17000 & 0 & 6,0 & 5,5 & \\
\hline & & TA & 17000 & 24 & 7,0 & 6,0 & \\
\hline & & 80 & 1500 & 0 & 16,75 & 14,0 & 1 \\
\hline & & 80 & 1500 & 24 & 11,25 & 10,0 & 3 \\
\hline & & 80 & 17000 & 0 & 6,75 & 5,5 & \\
\hline & & 80 & 17000 & 24 & 8,75 & 7,5 & \\
\hline \multirow{16}{*}{$\begin{array}{l}\frac{0}{\pi} \\
\frac{\pi}{0} \\
0 \\
0 \\
0\end{array}$} & \multirow{8}{*}{$\begin{array}{l}0^{m} \\
\tilde{\sigma}^{N} \\
\tilde{Z}^{\prime} \\
\Xi \\
\tilde{\delta}\end{array}$} & $\mathrm{TA}$ & 1500 & 0 & 1,75 & 1,5 & \\
\hline & & $\mathrm{TA}$ & 1500 & 24 & 9,5 & 4,5 & 2 \\
\hline & & $\mathrm{TA}$ & 17000 & 0 & 3,0 & 2,5 & \\
\hline & & $\mathrm{TA}$ & 17000 & 24 & 10,0 & 4,0 & 1 \\
\hline & & 80 & 1500 & 0 & 3,0 & 2,0 & \\
\hline & & 80 & 1500 & 24 & 9,5 & 4,5 & 2 \\
\hline & & 80 & 17000 & 0 & 7,25 & 1,5 & \\
\hline & & 80 & 17000 & 24 & 8,0 & 4,5 & 3 \\
\hline & \multirow{8}{*}{$\begin{array}{l}O^{m} \\
\tilde{\sigma}^{N} \\
\tilde{Z}^{2} \\
\tilde{D} \\
\tilde{\omega}\end{array}$} & TA & 1500 & 0 & 1,25 & 1,0 & 2 \\
\hline & & $\mathrm{TA}$ & 1500 & 24 & 1,25 & 1,0 & 2 \\
\hline & & $\mathrm{TA}$ & 17000 & 0 & 1,0 & 1,0 & \\
\hline & & $\mathrm{TA}$ & 17000 & 24 & 1,25 & 1,0 & 2 \\
\hline & & 80 & 1500 & 0 & 1,0 & 1,0 & \\
\hline & & 80 & 1500 & 24 & 1,5 & 1,5 & 1 \\
\hline & & 80 & 17000 & 0 & 0,75 & 0,5 & \\
\hline & & 80 & 17000 & 24 & 1,5 & 1,5 & 1 \\
\hline
\end{tabular}

bentoníticas [10-13].

A Fig. 2 apresenta as curvas de análises termodiferencial e termogravimétrica das argilas.

Nas curvas de ATD da argila chocolate foram observadas as seguintes transformações térmicas: grande banda endotérmica com máximo em $150{ }^{\circ} \mathrm{C}$, característica da presença de água livre e adsorvida; banda endotérmica com máximo em $572{ }^{\circ} \mathrm{C}$, característica da presença de hidroxilas da folha octaédrica; banda endotérmica com máximo em $864{ }^{\circ} \mathrm{C}$, característica da destruição do retículo cristalino e banda exotérmica com máximo em $903{ }^{\circ} \mathrm{C}$ da nucleação da mulita. Na curva ATG foram observados os eventos característicos da perda de água e perda de hidroxilas e uma perda total de massa de cerca de $26 \%$. Nas curvas de ATD da argila Brasgel PA foram observadas as seguintes transformações térmicas: grande banda endotérmica com máximo em $155^{\circ} \mathrm{C}$, característica da presença de água livre e adsorvida; banda endotérmica com máximo em $552{ }^{\circ} \mathrm{C}$, característica da presença de hidroxilas da folha octaédrica; banda endotérmica com máximo em $797^{\circ} \mathrm{C}$, característica da destruição do retículo cristalino e banda exotérmica com máximo em $899{ }^{\circ} \mathrm{C}$ da nucleação da mulita. Nas curvas de 
ATG foram observados os eventos característicos da perda de água e perda de hidroxilas e uma perda total de massa de cerca de $19 \%$. Nas curvas de ATD da argila closite $\mathrm{Na}^{+}$foram observadas as seguintes transformações térmicas: grande banda endotérmica com máximo em $159{ }^{\circ} \mathrm{C}$, característica da presença de água livre e adsorvida; banda endotérmica com máximo em $710{ }^{\circ} \mathrm{C}$, característica da presença de hidroxilas da folha octaédrica; banda endotérmica com máximo em $880{ }^{\circ} \mathrm{C}$, característica da destruição do retículo cristalino banda exotérmica com máximo em $910{ }^{\circ} \mathrm{C}$, característica da nucleação da mulita. Nas curvas de TG foram observados os eventos característicos da perda de água e perda de hidroxilas e uma perda total de massa de 17\%. Na Tabela IV estão apresentados os resultados de viscosidade aparente e plástica das dispersões obtidas com argila Cloisite $\mathrm{Na}^{+}$e seus métodos de preparação.

Como este padrão de viscosidades é típico dos fluídos de perfuração, foram utilizados as normas da Petrobrás para uma análise. Pode-se observar que muitas das amostras não atingiram os valores mínimos estipulados pela normalização da Petrobrás [14], fazendo com que seja necessária a classificação dos resultados como mais próximos da norma.

Tabela V- Distância interplanar basal $\left(\mathrm{d}_{001}\right)$ e intensidade das argilas organofilizadas.

[Table $V$ - Basal Interplanar distance $\left(d_{001}\right)$ and intensity of organoclay samples.]

\begin{tabular}{ccc}
\hline Amostra & $\begin{array}{c}\text { Distância } \\
\mathrm{d}_{001}(\AA)\end{array}$ & Intensidade (cps) \\
\hline Chocolate_HY_CD & $17,84 \pm 0,58$ & $596,18 \pm 136,14$ \\
Chocolate_HY_SD & $18,14 \pm 0,31$ & $722,45 \pm 110,65$ \\
Chocolate_WB_CD & $34,92 \pm 1,54$ & $733,68 \pm 102,30$ \\
Chocolate_WB_SD & $35,09 \pm 1,09$ & $817,62 \pm 88,27$ \\
Brasgel_HY & $19,13 \pm 0,39$ & $552,39 \pm 65,82$ \\
Brasgel_WB & $37,13 \pm 0,92$ & $890,14 \pm 91,08$ \\
Cloisite_HY & $17,24 \pm 0,36$ & $1038,66 \pm 131,25$ \\
Cloisite_WB & $23,98 \pm 0,72$ & $942,33 \pm 142,89$ \\
\hline
\end{tabular}

À direita da Tabela IV pode ser vista a classificação do resultado por ordem crescente de 1 até 4 . Vale salientar que viscosidades aparente e plástica com valores próximos indicaram um estado de floculação, mesmo que os valores estejam acima dos limites normalizados. Para amostras Brasgel e chocolate sem defloculante os melhores resultados de dispersão ocorreram nas amostras agitadas a 17000 rpm; para a amostra chocolate sem defloculante, praticamente não houve influência das variáveis de preparação [15]. O baixo desempenho da closite $\mathrm{Na}^{+}$dispersa em altas velocidades pode ser atribuído a um alto grau de delaminação das camadas e conseqüente dificuldade de reagrupamento em interações "face-to-edge". É importante considerar a presença dos cátions nas amostras de argila, sabendo-se que um sistema bentonita- $\mathrm{Na}^{+}$é altamente tixotrópico, em razão de uma espessa camada de água bem orientada em torno de cada lamela, daí as viscosidade elevadas do sistema com sódio como cátion interlamenlar, como no caso da closite $\mathrm{Na}^{+}$, Brasgel e chocolate com defloculante. Estes mesmos sistemas, na presença de $\mathrm{Ca}^{2+}$ e $\mathrm{Mg}^{2+}$, presentes também em todas as amostras, conforme Tabela III, e o $\mathrm{Na}^{+}$não interlamelar, sofrem sensível redução de viscosidade devido a reversibilidade da troca catiônica e a redução da camada de água fixada as lâminas de argila resultando, como no caso da chocolate sem defloculate, e mesmo da com defloculante em comparação com as demais argilas, em uma baixa viscosidade [15]. Foi também efetuada uma correlação entre o método de preparação da dispersão e a eficiência do processo de organofilização, como forma de se verificar os melhores resultados de reologia que corresponderiam aos melhores resultados de organofilização.

$\mathrm{Na}$ Tabela V estão apresentadas as distâncias interplanares basais com suas respectivas intensidades obtidas dos difratogramas das argilas organofílicas obtidas a partir das argilas estudadas tratadas com os tensoativos iônicos Praepagen HY e WB no teor de $60 \%$.

O controle de orgofilização por DRX está contido na Tabela V. O resultado médio da DRX, considerando-se apenas a expansão interplanar basal $\left(\mathrm{d}_{001}\right)$ e sua intensidade

Tabela VI - Perda de massa (\%) das argilas organofilizadas com tensoativo Praepagem HY e WB.

[Table VI - Weight loss (\%) of organoclays with surfactant Praepagem HY and WB.]

\begin{tabular}{cccccc}
\hline \multirow{2}{*}{ Argila } & Tensoativo & $\begin{array}{c}\text { Média } \\
\text { (Perda de massa) } \\
(\%)\end{array}$ & Desvio Padrão & $\begin{array}{c}\text { Mínimo } \\
(\%)\end{array}$ & $\begin{array}{c}\text { Máximo } \\
(\%)\end{array}$ \\
\hline \multirow{2}{*}{ Chocolate_CD } & HY & 28,12 & $\pm 1,10$ & 27,02 & 29,22 \\
& WB & 40,71 & $\pm 0,62$ & 40,08 & 41,33 \\
Chocolate_SD & HY & 28,12 & $\pm 0,89$ & 27,22 & 29,01 \\
& WB & 39,81 & $\pm 0,55$ & 39,26 & 40,36 \\
Brasgel PA & HY & 28,08 & $\pm 1,17$ & 26,90 & 29,25 \\
& WB & 40,91 & $\pm 0,51$ & 40,40 & 41,42 \\
Cloisite $\mathrm{Na}^{+}$ & HY & 27,58 & $\pm 0,68$ & 26,91 & 28,26 \\
& WB & 38,98 & $\pm 0,76$ & 38,22 & 39,75 \\
\hline
\end{tabular}


em função de todas as variáveis de processo considerada neste trabalho apresentam pequenas variações na distância interplanar $\left(\mathrm{d}_{001}\right)$. Analisando a argila Chocolate $\mathrm{CD}$, com o tensoativo Praepagem HY a distância interplanar media $\left(\mathrm{d}_{001}\right)$ é de $17,84(\AA) \pm 0,58$ e com intensidade média 596,18 (CPS) $\pm 136,14$, para a argila chocolate $\mathrm{SD}$, com o tensoativo Praepagem HY a distância interplanar media $\left(\mathrm{d}_{001}\right)$ é de $18,14(\AA) \pm 0,31$ e com intensidade média 722,45 (CPS) \pm 110,65 . Estes valores indicam que as amostras não tratadas com defloculante sódico foram capazes de realizar trocas catiônicas de maneira mais eficiente que as amostras não tratadas. As demais variáveis não produziram diferenças significativas com relação a distância interplanar e da intensidade do pico. Analisando agora a argila chocolate $\mathrm{CD}$, com o tensoativo Praepagem WB a distância interplanar media $\left(\mathrm{d}_{001}\right)$ é de $34,92(\AA) \pm 1,54$ e com intensidade média 733,68 (CPS) \pm 10230 , para a argila chocolate SD, com o tensoativo Praepagem WB a distância interplanar media $\left(\mathrm{d}_{001}\right)$ é de 35,09 $(\AA) \pm 1,09$ e com intensidade média 817,62 (CPS) $\pm 88,27$. Estes valores indicam novamente que as amostras não tratadas com defloculante sódico foram capazes de realizar trocas catiônicas de maneira mais eficiente que as amostras não tratadas. Como já dito as demais variáveis não produziram diferenças significativas de distância interplanar e intensidade. Para a argila Brasgel com o tensoativo Praepagem HY a distância interplanar media $\left(\mathrm{d}_{001}\right)$ é de $19,13(\AA) \pm 0,39$ e com intensidade média 552,39 (CPS) $\pm 65,82$. Para o tensoativo Praepagem WB a distância interplanar media $\left(\mathrm{d}_{001}\right)$ é de $37,13(\AA) \pm 0,92$ e com intensidade média 890,14 (CPS) $\pm 91,08$. Esses valores evidenciam como no caso da Chocolate que o modifica a posição do pico sendo as demais variáveis de poucas ou nenhuma influencia. Para a argila closite $\mathrm{Na}^{+}$ com o tensoativo Praepagem HY a distância interplanar media $\left(\mathrm{d}_{001}\right)$ é de $17,24(\AA) \pm 0,36$ e com intensidade média 1038,66 (CPS) $\pm 131,25$. Esses valores evidenciam a pequena variação da distância interplanar e da intensidade do pico de $17(\AA)$, não se podendo considerar as diferenças significativas em função das variáveis de processo envolvidas. Para o tensoativo Praepagem WB considerações semelhantes pode ser formuladas, tendo em vista que a distancia interplanar basal $\left(\mathrm{d}_{001}\right)$ média $23,98(\AA)$ $\pm 0,72$ e com intensidade médiam de 942,33 CPS $\pm 142,89$. Observa-se também que a dispersão de dados da distancia interplanar basal $\left(\mathrm{d}_{001}\right)$ é muito pequena e que a dispersão dos dados relativos a intensidade é pequena. As diferenças observadas entre as distâncias interplanares basais $\left(\mathrm{d}_{001}\right)$ entre os dois tensoativos deve-se aos diferentes tamanhos de suas moléculas e aos diferentes arranjos na galeria.

$\mathrm{Na}$ Tabela VI estão apresentados os resultados estatísticos dos termogramas das argilas organofílicas obtidas a partir das argilas estudas tratadas com o tensoativo iônico Praepagen HY e WB no teor de $60 \%$, com todas as variáveis de processo apresentadas na Tabela I

O controle de organofilização por análise termogravimétrica está contido na Tabela VI. Podese observar que as amostras organofilizadas em função das variáveis de processo consideradas neste trabalho apresentaram pequena variação na perda de massa que corresponde a quantidade de tensoativo intercalado.

Para amostra chocolate $\mathrm{CD}$ com o tensoativo $\mathrm{HY}$ o valor médio foi de $28,12 \% \pm 1,1$, este valor evidencia a pequena variação na quantidade de tensoativo intercalado em função de todas as variáveis de processo envolvidas neste trabalho. Para o tensoativo Preapagem WB considerações semelhantes podem ser formuladas tendo em vista que a quantidade de tensoativo adsorvido foi de $40,71 \% \pm 0,62$ este valor evidencia a pequena variação na quantidade de tensoativo intercalado em função de todas as variáveis de processo envolvidas neste trabalho. Para amostra chocolate SD com o tensoativo HY o valor médio foi de $28,12 \% \pm 0,89$, valor idêntico ao HY_CD. Para o tensoativo Preapagem WB observamos uma leve redução agora para o valor de $39,81 \% \pm 0,55$. Daí considerações semelhantes podem ser formuladas tendo em vista a pequena variação da quantidade de tensoativo trocado.

Para amostra Brasgel com o tensoativo HY o valor médio foi de $28,08 \% \pm 1,1$, este valor, novamente, evidencia a pequena variação na quantidade de tensoativo intercalado em função de todas as variáveis de processo envolvidas neste trabalho. Para o tensoativo Preapagem WB considerações semelhantes podem ser formuladas tendo em vista que a quantidade de tensoativo adsorvido foi de $40,91 \% \pm 0,51$ este valor evidencia a pequena variação na quantidade de tensoativo intercalado em função de todas as variáveis de processo envolvidas neste trabalho.

Para a Cloisite $\mathrm{Na}^{+} \mathrm{o}$ valor médio para o tensoativo Prepagem HY de $27,58 \% \pm 0,68$, este valor evidencia a pequena variação na quantidade de tensoativo intercalado em função de todas as variáveis de processo envolvidas neste trabalho. Para o tensoativo Preapagem WB considerações semelhantes podem ser formuladas tendo em vista que a quantidade de tensoativo adsorvido foi de $38,98 \% \pm 0,76$ este valor evidencia a pequena variação na quantidade de tensoativo intercalado em função de todas as variáveis de processo envolvidas neste trabalho.

Uma análise conjunta das Tabelas IV, V e VI evidencia que a viscosidades aparente e plástica, obtidas quando da dispersão das argilas que antecede ao processo de organofilização, não interferem nos resultados finais relativos a quantidade de tensoativos intercaladas sendo consideradas variáveis importantes o tipo de argila, o tipo de tensoativo e o tratamento com defloculante sódico. Deve ser considerado que alguns dos pontos considerados foram patenteados [16], os trabalhos resultaram de um projeto FINEP, cujo o titulo "Argilas organofílicas para uso como cargas nanométricas em matrizes poliméricas" desenvolvidos pela Oxiteno S.A Indústria e Comércio, UFCG. UFSCar e UNICAMPI. As simplificações operacionais no processo de organofilização, resultante deste trabalho, são costumeiramente utilizadas em nossos laboratórios, com resultados adequados, para incorporação em matrizes poliméricas e fluidos de perfuração base orgânicas. Os trabalhos de pesquisa considerando as 
variáveis de processos estão sendo estendidos para novas ocorrências de bentonitas situadas em Municípios de Cubatí e Pedra Lavrada, PB.

\section{CONCLUSÕES}

As variáveis consideradas pouco interferem no processo de organofilização, o que permitiu sua melhor operacionalidade industrial, podendo-se destacar os seguintes pontos: na preparação da dispersão que antecede o processo de organofilização, o uso de temperaturas ambientes, baixas velocidades de agitação da ordem de $1500 \mathrm{rpm}$, tempo de cura nulo, e quando ao processo de organofilização pode-se destacar os seguintes pontos: uso de temperaturas ambientes, baixas velocidades de agitação da ordem de $1500 \mathrm{rpm}$ e tempo de cura do processo de organofilização de $24 \mathrm{~h}$. Estas condições de processamento são mais simples de execução do ponto de vista industrial o que resulta em uma maior produtividade. Foram consideradas influentes as variáveis: tipo de argila, tipo de tensoativo e tratamento com defloculante sódico.

\section{REFERÊNCIAS}

[1] F. Bergaya, G. Lagaly. Appl. Clay Sci. 19 (2001) 1-3. [2]L. A. Ultracki, Clay-containing Polymeric Nanocomposites, Ed. Rapra Tecnology Ltd., UK 1 (2004) 84-85.

[3] L. B. Paiva, A. R. Morales, F. Valenzuela Diaz, Appl. Clay Sci. 20 (2008) 8-24.

[4] L. B. Paiva, A. R. Morales, F. Valenzuela Diaz, Cerâmica 54, 330 (2008) 213-226.

[5] H. S. Ferreira, Obtenção de argilas organofilicas purificadas para uso em fluidos de perfuração base óleo,
Diss. Mestrado, Programa de Pós Graduação em Ciência e Engenharia de Materiais, UFCG, PB (2005)1-78.

[6] M. D. Amir. Foster. Miner. 38, 994 (1953) 994-1006.

[7] I. F. Leite, A. P. S. Priscila, L. H. Carvalho, C. M. O. Raposo, O. M. L. Malta, S. M. L. Siva, J. Thermal Anal. Calorim. 100 (2010) 563-569.

[8] H. C. Ferreira, T. Chen, A. R. Zandonadi, P. S. Santos, Cerâmica 18 (1972) 333-339.

[9] H. S. Ferreira, R. R. Menezes, H. S. Ferreira, A. B. Martins, G. A. Neves, H. C. Ferreira, Cerâmica 54 (2008) 77-85.

[10] P. S. Santos, Ciência e tecnologia de argilas, $2^{a}$ Ed., Vol. 1, Edgard Blücher, S. Paulo, SP (1989) 338.

[11] L. V. Amorim, Melhoria, proteção e recuperação da reologia de fluidos hidroargilosos para uso na perfuração de poços de petróleo, Tese Dr., Engenharia de Processos/ CCT/UFCG, PB (2003) 234.

[12] L. F. A. Campos, Composições de argilas bentoniticas para utilização em fluidos de perfuração de poços de petróleo, Tese Dr., Engenharia de Processos/CCT/UFCG, PB (2007) 137.

[13] A. C. C. Esteves, A. B. Timmons, T. Trindade, Quím. Nova 27 (2004) 798-806.

[14] Petrobrás, Viscosificante para fluidos usados na exploração e produção de poços de petróleo e gás, Norma EP-1EP-00011-A, 2011A.

[15] S. Simon, D. L. Cerf, L. Picton, G. Muller, Colloids and Surfaces A: Physicochem. Eng. Aspects 203 (2002) 77-86.

[16] A. L. C. Silva, D. L. Costa, G. A. Neves, H. C. Ferreira, H. S. Ferreira, R. F. Cossiello, T. J. A. Melo, Processo de obtenção de filossilicatos organofilicos, filossilicatos organofilicos e seus usos, PI 0901329-6 A2, INPI (2011).

(Rec.27/04/2012, Ac. 08/06/2012) 